Article

\title{
Comparative Analysis of Biologically Relevant Response Curves in Gene Expression Experiments: Heteromorphy, Heterochrony, and Heterometry
}

\author{
Stuart G. Baker \\ Biometry Research Group, National Cancer Institute, Bethesda, MD 20872, USA; \\ E-Mail:sb16i@nih.gov
}

Received: 20 December 2013; in revised form: 7 February 2014 / Accepted: 11 February 2014 /

Published: 14 February 2014

\begin{abstract}
To gain biological insights, investigators sometimes compare sequences of gene expression measurements under two scenarios (such as two drugs or species). For this situation, we developed an algorithm to fit, identify, and compare biologically relevant response curves in terms of heteromorphy (different curves), heterochrony (different transition times), and heterometry (different magnitudes). The curves are flat, linear, sigmoid, hockey-stick (sigmoid missing a steady state), transient (sigmoid missing two steady states), impulse (with peak or trough), step (with intermediate-level plateau), impulse+ (impulse with an extra parameter), step+ (step with an extra parameter), further characterized by upward or downward trend. To reduce overfitting, we fit the curves to every other response, evaluated the fit in the remaining responses, and identified the most parsimonious curves that yielded a good fit. We measured goodness of fit using a statistic comparable over different genes, namely the square root of the mean squared prediction error as a percentage of the range of responses, which we call the relative prediction error (RPE). We illustrated the algorithm using data on gene expression at 14 times in the embryonic development in two species of frogs. Software written in Mathematica is freely available.
\end{abstract}

Keywords: double sigmoid; microarray; relative prediction error; sigmoid; time series 


\section{Introduction}

Many gene expression experiments involve serial measurements in response to a varying condition, such as temperature, oxygen availability, time, drug concentration, levels of pollutants, and exposures to ultraviolet light. Often investigators want to compare the time varying response between two scenarios, such as two species or two drugs. For this comparative analysis, we developed an algorithm to fit biologically relevant curves to serial response measurements from each gene, identify pairs of curves that fit well, and compare these curves under the two scenarios in terms of heteromorphy (different curves), heterochrony (different transition times) and heterometry (different magnitudes). In the context of ontogeny, Yanai et al. [1] introduced the concepts of heteromorphy and heterochrony in gene expression curves as analogs to tissue-level heteromorphy (different sizes of developing organs) and heterochrony (movement of modules in anatomy and physiology). In other comparative gene expression settings heteromorphy and heterochrony in gene expression curves can also provide insight into biological processes. The purpose of this methodology is to compare gene expression patterns in two settings, as guided by biologically relevant models. To simplify this discussion, we consider time as the time varying condition.

The fitting part of the algorithm involves the following models: flat, linear, sigmoid, double sigmoid [2], and generalized double sigmoid [3]. The flat and linear models yield flat and linear response curves, respectively. The sigmoid model yields a sigmoid curve (two steady states with an intermediate transition following a logistic function), a hockey stick curve (sigmoid curve missing one steady state) or a transition curve (sigmoid missing two steady states). The double sigmoid is the product of two sigmoid models; it yields an impulse curve (with a peak or trough) or step curve (with an intermediate-level plateau). The generalized double sigmoid curve adds an additional parameter to the double sigmoid model and yields analogous impulse + curve or step + curves. We also characterized all the curves, except for flat, as either trending upward or downward or having a downward or upward impulse.

The aforementioned response curves are biologically relevant, as opposed to polynomial curves of degree two or greater, which generally have little biological basis. Flat curves represent a steady state. Linear curves represent the constant addition or subtraction of reacting components. Sigmoid curves model the addition or subtraction of reacting components from one steady state to another steady state. Sigmoid curves also arise in transcription factor binding [4,5]. Impulse and impulse + curves represent a temporary increase or decrease in reacting components that resolves into a new steady state [1]. Step and step + curves represent an intermediate-level steady state amid a monotonically increasing or decreasing number of reacting components.

Although there is a large literature on the fitting of response curves to sequential gene expression measurements in dose-response and short time series studies [6-10], there has been little work on the comparative analysis of response curves. A notable exception is Sivriver, et al. [3] who fit and compared generalized double sigmoid response curves under two stimuli. A major concern of Sivriver et al. [3] was overfitting. Overfitting means that a model has so many parameters relative to time points that chance deviations from the true model strongly influence the model fit and lead to poor predictions at time points not used in model fitting. Here overfitting is particularly a concern for two reasons. First the generalized double sigmoid and double sigmoid models have a large number of parameters relative 
to the number of time points. Second the investigation of over ten thousands genes implies a much higher probability of substantial chance deviations in the response curves for some genes than if only a few genes were studied. Sivriver, et al. [3] tackled the problem of overfitting by pooling data from multiple genes with similar generalized double sigmoid response curves. Because we focus on heterochrony and heterometry, which would be diluted by pooling, we developed a different approach to reduce overfitting that does not involve pooling. In our approach we evaluated model fits at different times from those used to fit the model. In particular we fit biologically relevant curves to every other response (first, third, fifth, ...) and evaluated the fits at the remaining responses (second, fourth, sixth, ...), providing an empirical investigation of model fit. Because we used seven points for model fitting and seven points for model evaluation, we needed at least 14 points to adequately fit and evaluate the generalized double sigmoid model, which has seven parameters.

For illustration, we applied our algorithm to mean gene expression levels (averaged over three technical replicates and three specimens) for 11,299 genes at 14 development times in two species of frogs, X.laevis and X.tropicalis. [1]. We found some interesting examples of heteromorphy and heterochrony that will hopefully spur new research. However, the main contribution of this paper is a method for identifying the most interesting changes in pairs of biologically relevant shapes for gene expression curves in comparative studies.

\section{Identifying Biologically Relevant Response Curves that Fit Well}

As noted by Forster [11] standard methods of model selection (such as likelihood ratio tests, the Akaike Information Criterion (AIC), the Bayesian Information Criterion, and Minimum Description Length [11-13]) minimize the discrepancy between predicted and observed results at the same time points used to fit the model. In the spirit of Forster [11] and with the emphasis on reducing overfitting, we were instead interested in minimizing the discrepancy between predicted and observed results at different time points than used to fit the model. Similarly, Chechik and Koller [2] evaluated double sigmoid fits at a single point that was left out of the fitting procedure. We considered every other point as left-out in order to examine discrepancy between observed and predicted results over the entire range of times.

Consider a single gene. Let $y_{j}$ denote the $j^{\text {th }}$ observed response and $x_{j}$ denote the $j^{\text {th }}$ observed time. We fit the model to responses $\left\{y_{1}, y_{3}, y_{5}, y_{7}, y_{9}, y_{11}, y_{13}\right\}$ at times $\left\{x_{1}, x_{3}, x_{5}, x_{7}, x_{9}, x_{11}, x_{13}\right\}$. We call $\left\{\left(x_{1}, y_{1}\right),\left(x_{3}, y_{3}\right),\left(x_{5}, y_{5}\right),\left(x_{7}, y_{7}\right),\left(x_{9}, y_{9}\right),\left(x_{11}, y_{11}\right)\right\}$ the fitted points. We evaluate the model at $\left\{\left(x_{2}, y_{2}\right)\right.$, $\left.\left(x_{4}, y_{4}\right),\left(x_{6}, y_{6}\right),\left(x_{8}, y_{8}\right),\left(x_{10}, y_{10}\right),\left(x_{12}, y_{12}\right)\right\}$, which we call the evaluation points Let $\left\{f\left(x_{2}\right), f\left(x_{4}\right), f\left(x_{6}\right)\right.$, $\left.f\left(x_{8}\right), f\left(x_{10}\right), f\left(x_{12}\right), f\left(x_{14}\right)\right\}$ denote the predicted responses of a particular model corresponding to the evaluation points.

We needed a measure of how well the predicted responses fit the observed evaluation points. One measure considered was the mean squared error (MSE). The problem with using MSE is that it depends on the absolute sizes of responses, so two genes could have the same MSE's for comparing predicted and observed responses, yet visually one may fit well and the other fit poorly. To circumvent this problem we introduced the Relative Prediction Error (RPE), which is the square root of the MSE of the predicted response divided by the difference between the largest and smallest predicted responses, expressed as a percentage. The reason for using the square root is to put the measure on the 
same scale as the responses, analogous to using a standard deviation instead of a variance. The reason for dividing by the range of responses is to make small deviations between predicted and observed response relative to the entire shape of the curve, which leads to a visually satisfying measure. Let $J=\{2,4,6,8,10,12,14\}$ index the evaluation points. Mathematically we write RPE for our situation with 14 time points as

$$
\mathrm{RPE}=\left[\Sigma_{j \text { in } J}\left\{y_{j}-f\left(x_{j}\right)\right\}^{2} / 7\right]^{1 / 2} /\left[\max _{j \text { in } J}\left\{f\left(x_{j}\right)\right\}-\min _{j \text { in } J}\left\{f\left(x_{j}\right)\right\}\right]
$$

The formula for RPE can be readily modified for more than 14 points. Based on a visual inspection of curves with different values of RPE, we decided that a threshold of $10 \%$ was a reasonable indicator of a good fit. To put the idea of a threshold RPE into perspective, note that a likelihood ratio test comparing observed and fitted counts typically also involves a threshold, namely a 5\% type I error.

When comparing predicted and observed results at different time points than used to fit the model, Forster [11] evaluated the fit of the model without considering the complexity of the model. A rationale is that an evaluation at different time points than used for fitting inherently penalizes for complexity that leads to overfitting. Nevertheless, visual inspection suggests that parsimony is desirable for characterizing curves based on their fits to the evaluation points. For characterizing parsimony using the evaluation points we allow a 5\% leeway in terms of RPE for a curve with fewer parameters than the curve with smallest RPE. In other words, if a response curve has fewer parameters than the response curve with smallest RPE, we prefer the response curve with fewer parameters if its RPE is less than or equal to the smallest RPE plus 5\% We chose the value of 5\% based on visual inspection of many curves.

To introduce the curve selection algorithm, consider the following two hypothetical examples for a single gene. In the first example, suppose the RPE's for flat, lineU, sigmoidU, impulseU, and impulse $+\mathrm{U}$ curves are $30 \%, 12 \%, 11 \%, 8 \%$, and $9 \%$, respectively (as explained in the next section, the "U" designates upward trend).

Step 1. In this first example the best fitting curve is impulseU because it has the smallest RPE, namely $8 \%$. Because this RPE of $8 \%$ is less than or equal to the $10 \%$ RPE threshold for a good fit, we consider impulseU a good fit and investigate a more parsimonious curve in Step 2. Otherwise, if this RPE were greater than $10 \%$, we would not report a response curve for this gene.

Step 2. In this first example, lineU and sigmoidU satisfy the 5\% RPE leeway requirement, both having an RPE $\leq 8 \%+5 \%=13 \%$. Because lineU has fewer parameters than sigmoidU, we select lineU as the reported response curve.

In the second hypothetical example, suppose the RPE's for flat, lineU, sigmoidU, impulseU, and impulse+U curves are $30 \%, 22 \%, 14 \%, 8 \%, 9 \%$, respectively.

Step 1. In this second example, the best fitting curve is impulseU because it has the smallest RPE, namely $8 \%$. Because it is a good fit with RPE $<10 \%$, we investigate a more parsimonious model in Step 2.

Step 2. In this second example, no curve with fewer parameters than impulseU satisfies the 5\% RPE leeway requirement. Therefore we select impulseU as the reported response curve. However, for purposes of comparison, we identify the curve with the next fewest parameters than impulseU, namely sigmoidU. 
We formalize the curve selection algorithm as follows.

Step 1. Let Curve A denote the response curve with the smallest RPE, which we denote $\mathrm{RPE}_{\mathrm{A}}$. In the first example Curve $\mathrm{A}$ is impulseU. If $\mathrm{RPE}_{\mathrm{A}}>10 \%$, report no curve; otherwise proceed to Step 2.

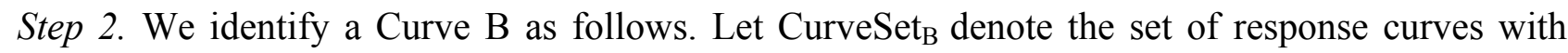
fewer parameters than Curve A. In the first example CurveSet $_{B}=\{$ flat, lineU, sigmoidU $\}$. Let CurveSubset $_{B}$ denote a subset of response curves in CurveSet $_{B}$ such that $R P E \leq \mathrm{RPE}_{\mathrm{A}}+5 \%$. In the

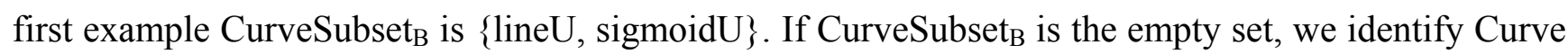
$\mathrm{B}$ as the curve with the most parameters in CurveSet $_{\mathrm{B}}$ (sigmoidU in the second example) but select Curve $A$ as the reported curve. If CurveSubset ${ }_{B}$ is not empty we identify Curve B as the curve in CurveSubset $_{B}$ with the fewest parameters (lineU in the first example) and select Curve B as the reported curve.

When we report a pair of response curves for a gene, we require that each response curve in the pair yield a good fit to the data with $\mathrm{RPE}_{\mathrm{A}} \leq 10 \%$. The curve reported for each gene in the pair is either Curve A or Curve B, whichever was selected via the curve selection algorithm.

In our application to frog data, the 5\% RPE leeway agreed well with the sign of the change in AIC, where $\mathrm{AIC}=7 \log \left[\Sigma_{j \text { in } J}\left\{y_{j}-f\left(x_{j}\right)\right\}^{2} / 7\right]+2 \times$ (number of parameters). Although this is a non-standard use of AIC because it applies to evaluation points instead of fitted points, it is still instructive. Figure 1 plots points for genes with good fitting models in both species of frogs. The points labeled Curve A (Curve B) selected correspond to reporting Curve A (Curve B) in the curve selection algorithm. Most Curve $A$ selected points, which require $\mathrm{RPE}_{\mathrm{A}}-\mathrm{RPE}_{\mathrm{B}}>5 \%$, correspond to $\mathrm{AIC}_{\mathrm{A}}-\mathrm{AIC}_{\mathrm{B}}>0$ (the upper right quadrant). Most Curve $B$ selected points, which require $\mathrm{RPE}_{\mathrm{A}}-\mathrm{RPE}_{\mathrm{B}} \leq 5 \%$, correspond to $\mathrm{AIC}_{\mathrm{A}}-\mathrm{AIC}_{\mathrm{B}} \leq 0$ (the lower left quadrant).

Figure 1. Comparison of a change in relative prediction error (RPE) with a change in Akaike Information Criterion (AIC) among response curve pairs. The red points corresponding to Curve $A$ require $\mathrm{RPE}_{\mathrm{A}}-\mathrm{RPE}_{\mathrm{B}} \leq 5 \%$ (so are above the horizontal 5\% line) The green points corresponding to Curve $\mathrm{B}$ require $\mathrm{RPE}_{\mathrm{A}}-\mathrm{RPE}_{\mathrm{B}} \leq 5 \%$ (so are below the horizontal $5 \%$ line). A value of $\mathrm{AIC}_{\mathrm{A}}-\mathrm{AIC}_{\mathrm{B}} \leq 0$ (so on the left of vertical line) would indicate selection of Curve B.

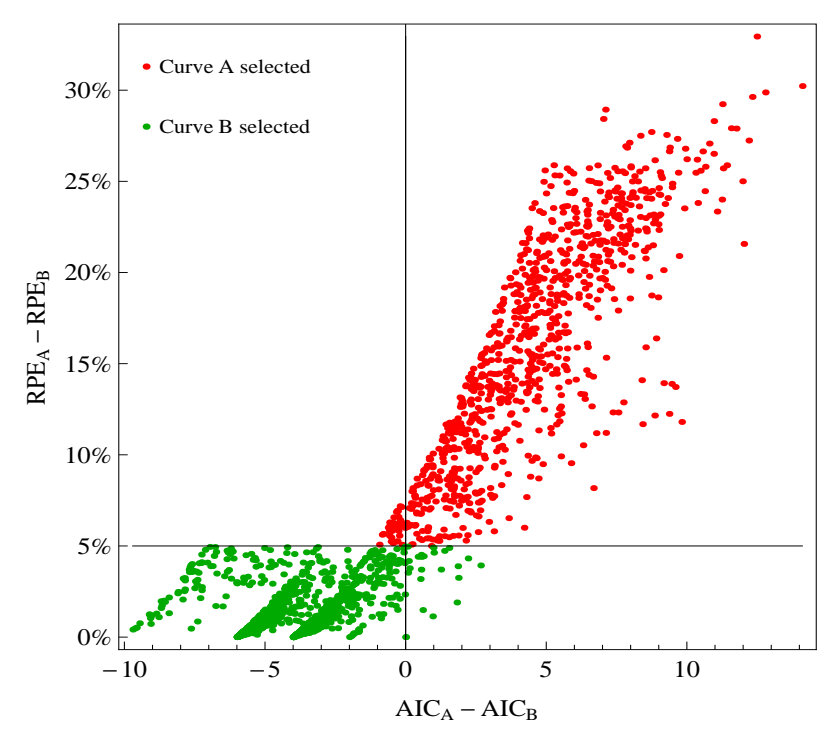




\section{Fitting Algorithms}

We fit all models using iteratively reweighted least squares with modifications to incorporate starting values. Let $x$ denote the varying condition, such as time. We discuss the formulas and fitting of each biologically relevant response curves in turn.

\subsection{Flat}

The flat curve has equation $\mathrm{f}_{\mathrm{FLA}}(x)=\alpha_{\mathrm{FLA}}$.

\subsection{Linear}

The linear curve has equation $\mathrm{f}_{\mathrm{LIN}}(x)=\alpha_{\mathrm{LIN}}+\beta_{\mathrm{LIN}} \cdot x$, for $\beta_{\mathrm{LIN}} \neq 0$. Letting $\mathrm{b}_{\text {LIN }}$ denote the estimate of $\beta_{\text {LIN }}$, we designated the linear model as lineD if $\mathrm{b}_{\text {LIN }}<0$ and lineU if $\mathrm{b}_{\text {LIN }}>0$, where $\mathrm{D}$ stands for downward and $\mathrm{U}$ stands for upward.

\subsection{Sigmoid}

The sigmoid curve starts with a steady state and then monotonically increases or decreases and finishes with another steady state (Figure 1). For flexibility, we fit one of two versions of the sigmoid model, depending on the estimated slope of the linear model,

$$
\mathrm{f}_{\mathrm{SIG}}(x)=\left\{\begin{array}{l}
\alpha_{\mathrm{SIG}}+\left(\gamma_{\mathrm{SIG}}-\alpha_{\mathrm{SIG}}\right) \operatorname{expit}\left\{\beta_{\mathrm{SIG}}\left(x-\delta_{\mathrm{SIG}}\right)\right\}, \text { if } \mathrm{b}_{\mathrm{LIN}}>0 \\
\alpha_{\mathrm{SIG}}+\left(\gamma_{\mathrm{SIG}}-\alpha_{\mathrm{SIG}}\right) \operatorname{expit}\left\{\beta_{\mathrm{SIG}}\left(\delta_{\mathrm{SIG}}-x\right)\right\}, \text { if } \mathrm{b}_{\mathrm{LIN}}<0
\end{array}\right.
$$

where expit $(x)=\exp (x) /\{1+\exp (x)\}$. The parameters $\alpha_{\mathrm{SIG}}$ and $\gamma_{\mathrm{SIG}}$ specify levels of the steady states. The parameter $\delta_{\mathrm{SIG}}$ is the horizontal point corresponding to the maximum slope, $\beta_{\mathrm{SIG}}$, between the steady states. The sign of $b_{\text {LIN }}$ is not always a reliable guide to the trend of the sigmoid curve, which we determine by simply comparing the first and last points on the sigmoid curve. We designated the downward and upward trending sigmoid curves as sigmoidD and sigmoidU, respectively.

\subsection{Hockey Stick}

The hockey stick curve is a sigmoid curve that is missing one steady state. We identified a steady state in a sigmoid curve as a slope at the beginning or the end of the curve that is less than or equal to 0.10 , a value chosen based on visual inspection. We designated the downward and upward trending hockey-stick curves as hockeyD and hockeyU, respectively.

\subsection{Transition}

A transition curve is a sigmoid curve that is missing two steady states, leaving only the transition region between the missing steady states. We designated the downward and upward trending transition curves as transitionD and transitionU, respectively. 


\subsection{Impulse}

The impulse curve is one type of curve (along with the sigmoid and step curves) arising from the double sigmoid model. For flexibility, we fit one of two versions of the double sigmoid model, depending on the estimated slope of the linear model,

$$
\begin{aligned}
& \left(1 / \beta_{\mathrm{dbs}}\right) \times\left[\alpha_{\mathrm{dbs}}+\left(\beta_{\mathrm{dbs}}-\alpha_{\mathrm{dbs}}\right) \operatorname{expit}\left\{\epsilon_{\mathrm{dbs}}\left(x-\delta_{\mathrm{dbs}}\right)\right]\right. \\
& \times\left[\gamma_{\mathrm{dbs}}+\left(\beta_{\mathrm{dbs}}-\gamma_{\mathrm{dbs}}\right) \operatorname{expit}\left\{-\epsilon_{\mathrm{dbs}}\left(x-\emptyset_{\mathrm{dbs}}\right)\right\}\right], \text { if } \mathrm{b}_{\mathrm{LIN}}>0 \\
& \mathrm{f}_{\mathrm{DBS}}(x)=\{ \\
& \left(1 / \beta_{\mathrm{dbs}}\right) \times\left[\alpha_{\mathrm{dbs}}+\left(\beta_{\mathrm{dbs}}-\alpha_{\mathrm{dbs}}\right) \operatorname{expit}\left\{\epsilon_{\mathrm{dbs}}\left(\delta_{\mathrm{dbs}}-x\right)\right\}\right] \\
& \times\left[\gamma_{\mathrm{dbs}}+\left(\beta_{\mathrm{dbs}}-\gamma_{\mathrm{dbs}}\right) \operatorname{expit}\left\{-\epsilon_{\mathrm{dbs}}\left(\varnothing_{\mathrm{dbs}}-x\right)\right\}\right], \text { if } \mathrm{b}_{\mathrm{LIN}}<0
\end{aligned}
$$

To avoid numerical problems, we only fit the double sigmoid model if the RPE of the sigmoid model was larger than the RPE of the linear model. Starting values come from the fit of the sigmoid model, namely $\alpha_{\mathrm{dbs}}=a_{\mathrm{sig}}, \beta_{\mathrm{dbs}}=\left(a_{\mathrm{sig}}+g_{\mathrm{sig}}\right) / 2, \gamma_{\mathrm{dbs}}=g_{\mathrm{sig}}, \delta_{\mathrm{dbs}}=\delta_{\mathrm{sig}}, \epsilon_{\mathrm{dbs}}=0$, and $\emptyset_{\mathrm{dbs}}=0$, where $a_{\mathrm{sig}}$ and $g_{\text {sig }}$ are the estimates of $\mathrm{d}_{\text {sig }}$ and $\gamma_{\text {sig }}$, respectively.

The impulse curve has a peak or trough between steady states (Figure 2) although sometimes the steady states are missing. The parameters $d_{\mathrm{dbs}}$ and $\gamma_{\mathrm{dbs}}$ correspond to levels of the flat sections. For example with $b_{L I N}>0$ and $\epsilon_{\mathrm{dbs}}>0, \mathrm{f}_{\mathrm{DBS}}(x)$ is approximately $\left(1 / \beta_{\mathrm{dbs}}\right) \times \alpha_{\mathrm{dbs}} \times \beta_{\mathrm{dbs}}=\alpha_{\mathrm{dbs}}$ for small values of $x$ and approximately $\left(1 / \beta_{\mathrm{dbs}}\right) \times \beta_{\mathrm{dbs}} \times \gamma_{\mathrm{dbs}}=\gamma_{\mathrm{dbs}}$ for large values of $x$. The parameter $\beta_{\mathrm{dbs}}$ determines the level of the impulse. The parameter $\epsilon_{\mathrm{dbs}}$, which appears in each sigmoid factor, determines the slope of the peak or trough. Mathematically, we identified the impulse curve as a double sigmoid curve in which the minimum or maximum did not occur at the endpoints. We designated an impulse curve with a trough and peak as impulseD and impulseU, respectively.

\subsection{Step}

The step curve is a double sigmoid curve with an intermediate plateau between steady states, although sometimes the steady states are missing. Mathematically, we identified the step curve as a double sigmoid curve in which both the minimum and maximum occur at the endpoints. Although this identification procedure would also detect a sigmoid curve, the sigmoid curve is preferentially selected via the sigmoid model. We designated the downward and upward trending step curves as stepD and stepU, respectively.

\subsection{Impulse+}

Sivriver, et al. [2] generalized the impulse double sigmoid model to allow for different slopes before and after the peak or trough of an impulse curve. We call the analog of the impulse curve for the generalized double sigmoid model the impulse+ curve. We parameterized the generalized double sigmoid by multiplying $-\epsilon_{\mathrm{dbs}}$ in Equation (3) by an additional parameter $\lambda_{\mathrm{dbs}}$. We used the parameter estimates from the impulse curve as starting values with $\lambda_{\mathrm{dbs}}=0$. We identified the impulse + curve as a generalized double sigmoid curve in which the minimum or maximum was not at the endpoints. We designated an impulse + curve with a trough and peak as impulse $+\mathrm{D}$ and impulse $+\mathrm{U}$, respectively. 


\subsection{Step +}

We identified the step+ curve as a generalized double sigmoid curve in which both the minimum and maximum occur at the endpoints. We designated the downward and upward trending step + curves as step $+\mathrm{D}$ and step $+\mathrm{U}$, respectively.

\section{Measuring Heterometry and Heterochrony}

\subsection{Heterometry}

We measured heterometry (HM) as the mean vertical difference between response curves expressed as a percentage of the vertical response range. We computed HM based on the following points on the response curves: (i) any point on the flat curve; (ii) the endpoints for linear, transient, hockey, sigmoid, or step and step+, curves; and (iii) the endpoints and the point at the peak or trough for impulse and impulse + curves. For Table 1, our indicator of heterometry was $\mathrm{HM} \leq 10 \%$.

Table 1. Response curve pairs with at least five counts.

\begin{tabular}{|l|l|l|l|l|l|}
\hline X.laevis & X.tropicalis & Total & $\begin{array}{l}\text { Heterochrony } \\
\text { only }\end{array}$ & $\begin{array}{l}\text { Heterometry } \\
\text { Only }\end{array}$ & $\begin{array}{l}\text { Heterochrony and } \\
\text { heterometry }\end{array}$ \\
\hline sigmoidU & sigmoidU & 694 & 18 & 347 & 70 \\
lineU & sigmoidU & 146 & 0 & 0 & 0 \\
sigmoidU & hockeyU & 73 & 0 & 0 & 0 \\
sigmoidD & sigmoidD & 48 & 3 & 22 & 0 \\
lineU & lineU & 47 & 0 & 39 & 0 \\
hockeyU & hockeyU & 30 & 0 & 18 & 1 \\
sigmoidU & lineU & 20 & 0 & 0 & 0 \\
lineU & hockeyU & 14 & 0 & 0 & 0 \\
hockeyU & sigmoidU & 13 & 0 & 0 & 0 \\
sigmoidU & impulseD & 9 & 0 & 0 & 0 \\
impulseD & sigmoidU & 8 & 0 & 0 & 0 \\
sigmoidD & lineD & 8 & 0 & 0 & 0 \\
sigmoidD & hockeyD & 5 & 0 & 0 & 0 \\
\hline
\end{tabular}

\subsection{Heterochrony}

We measured heterochrony ( $\mathrm{HC}$ ) as the mean horizontal difference between response curves as a percentage of the horizontal response range. We computed $\mathrm{HC}$ based on the following points on the response curves: (i) the horizontal point at the maximum absolute value of slope for sigmoid, hockey, transition, step, and step+ curves; and (ii) the horizontal point corresponding to the peak or trough of the impulse and impulse+ curves. We did not compute $\mathrm{HC}$ for flat or linear curves. For Table 1, our indicator of heterochrony was $\mathrm{HC} \leq 10 \%$.

\section{Results}

Of the 11,299 genes in the frog data, $10 \%$ of the response curves were good fits in both species of frogs (and used for the analysis) and 45\% were good fits in only one species of frog. Table 1 shows the distribution of the reported response curve pairs with at least five counts. The sigmoid curve 
predominated along with the closely related hockey-stick curve, but with relatively few impulse curves. Importantly, the algorithm did not select any transition, impulse + , or step + curves as best fitting with good fits to the data. The predominance of heterometry of heterochrony in Table 1 confirms earlier less formal investigations [1]. By examining lists of genes corresponding to the model pairs with nonzero counts in Tables $2-5$ and inspecting the fits with figures like Figures 2-5, investigators may be able to gain more insight into differences between the development of X.laevis and X.tropicalis. Of particular note are examples of heteromorphy in which the pair of curves trended in opposite directions (Tables 3 and 4).

Figure 2. Example of sigmoid curves for one gene pair. X.laevis and X.tropicalis are two species of frogs. The red points denoted "fitted" were used for model fitting. The black points denoted "evaluation" were used for model evaluation and computation of RPE. The curves with blue and green labels and lines are the reported curves (Curve A or Curve B, whichever was selected). The curves with orange labels and lines are included for comparison (Curve A or Curve B, whichever was not selected). HC and HM are measures of heterochrony and heterometry, respectively.
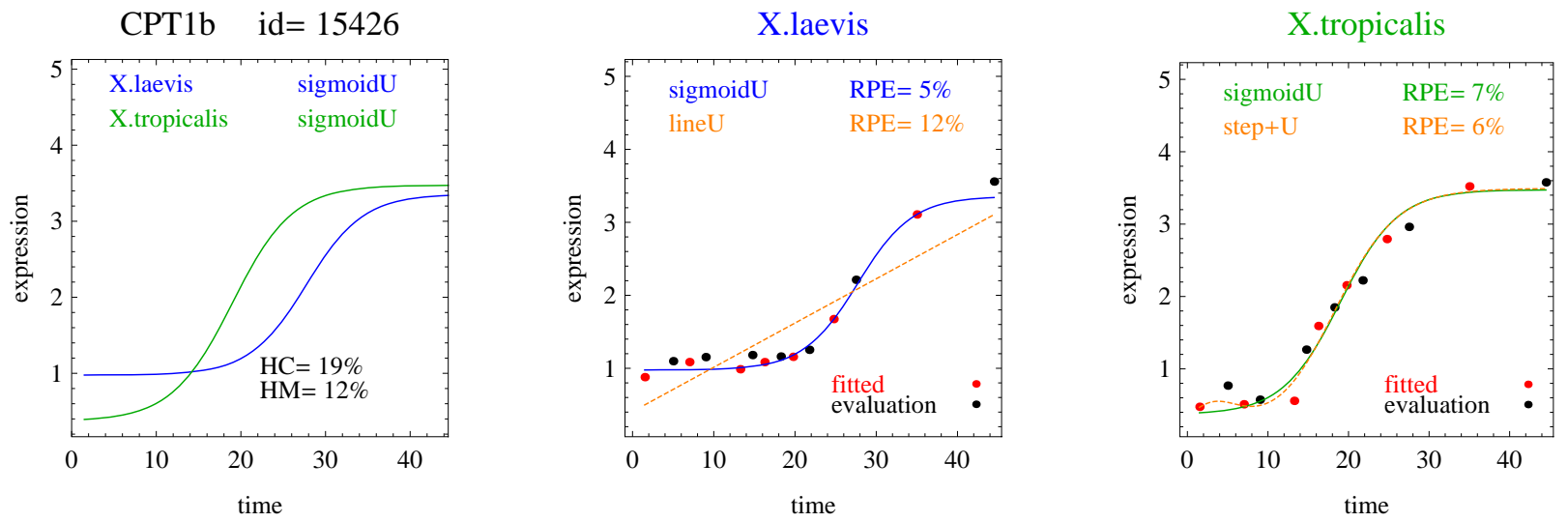

Figure 3. Example of impulse curves for one gene pair.
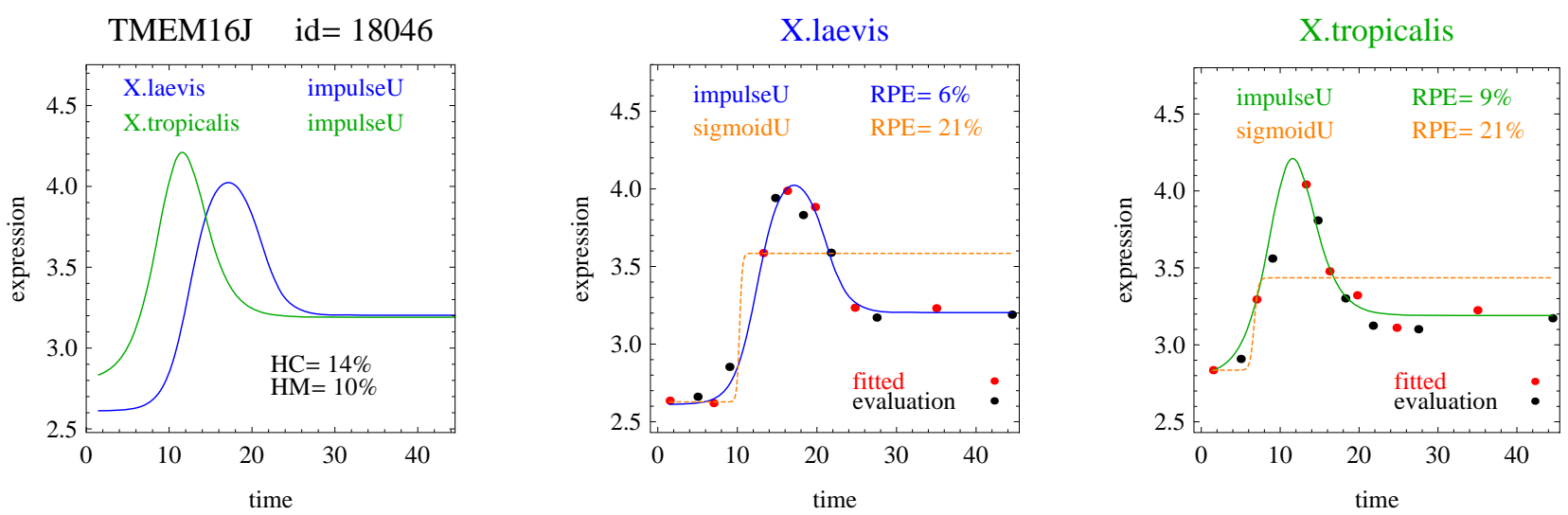
Figure 4. Example of a hockey stick and line for one gene pair.
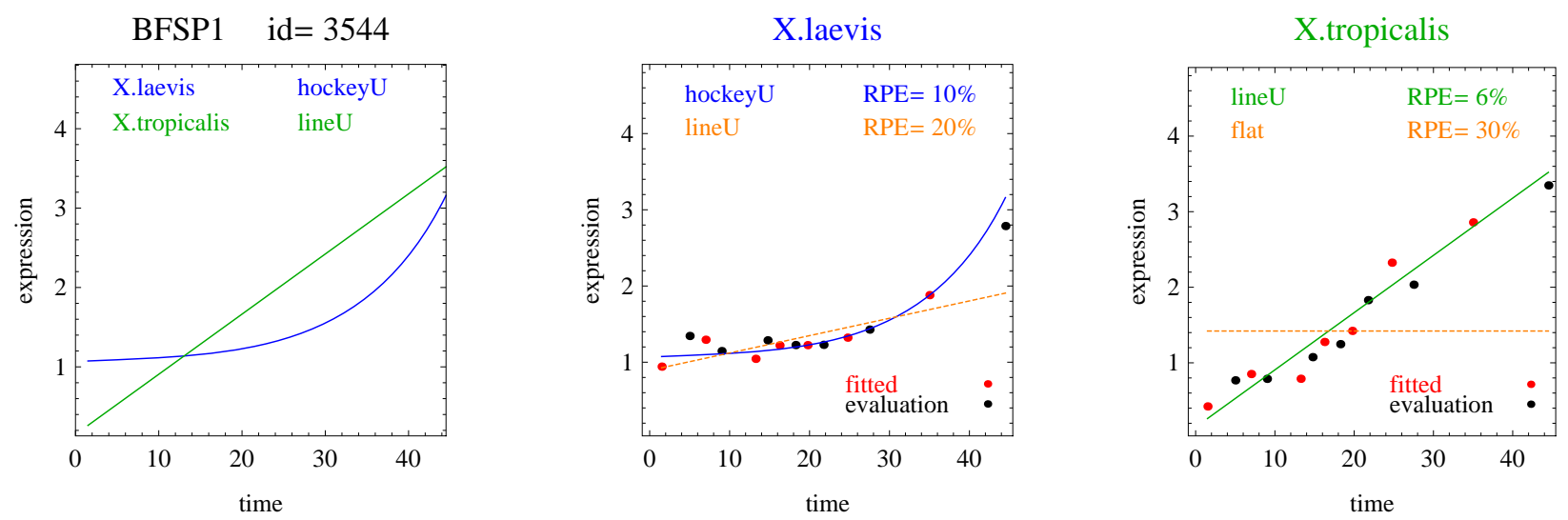

Figure 5. Example of a step curve and line for one gene pair.
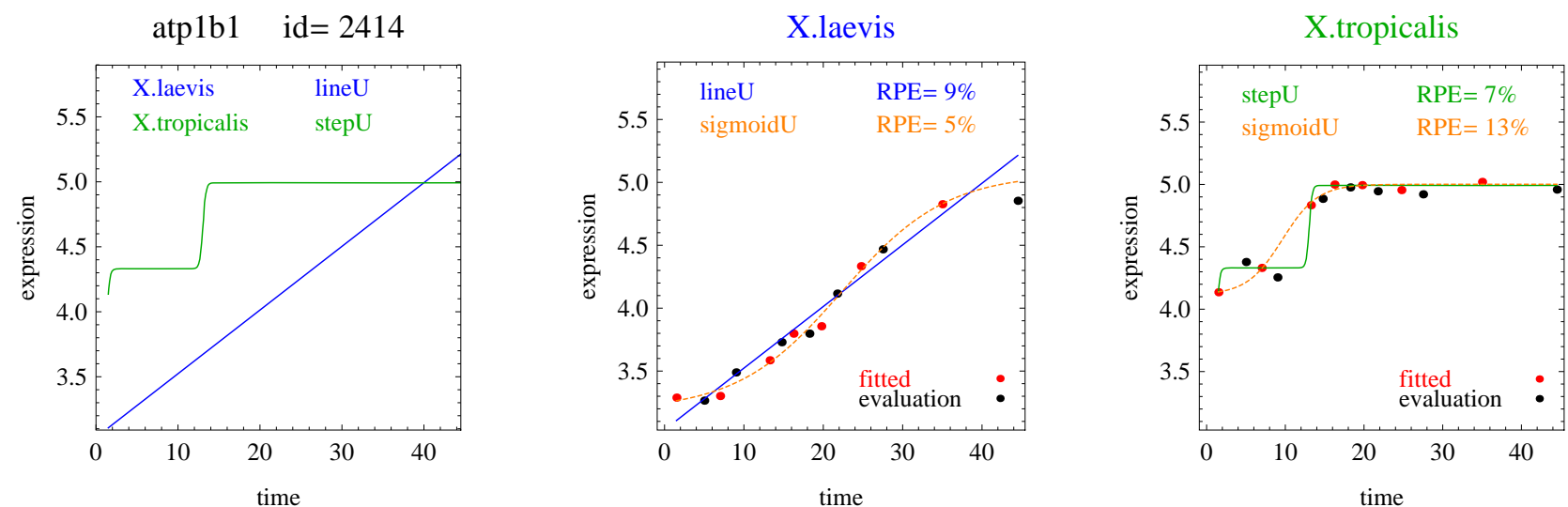

Table 2. Counts for response curve pairs with downward trends for both X.laevis and X.tropicalis. The total number is 68 .

\begin{tabular}{|l|l|l|l|l|l|l|l|l|l|}
\hline & \multicolumn{9}{|c|}{ X.tropicalis } \\
\hline X.laevis & flat & lineD & tranD & hocD & sigD & impD & stepD & imp+D & step + D \\
\hline Flat & 0 & 0 & 0 & 0 & 0 & 0 & 0 & 0 & 0 \\
\hline lineD & 0 & 3 & 0 & 0 & 1 & 0 & 0 & 0 & 0 \\
\hline tranD & 0 & 0 & 0 & 0 & 0 & 0 & 0 & 0 & 0 \\
\hline hocD & 0 & 0 & 0 & 0 & 0 & 0 & 0 & 0 & 0 \\
\hline sigD & 0 & 8 & 0 & 5 & 48 & 1 & 0 & 0 & 0 \\
\hline impD & 0 & 0 & 0 & 0 & 0 & 2 & 0 & 0 & 0 \\
\hline stepD & 0 & 0 & 0 & 0 & 0 & 0 & 0 & 0 & 0 \\
\hline impD+ & 0 & 0 & 0 & 0 & 0 & 0 & 0 & 0 & 0 \\
\hline stepD+ & 0 & 0 & 0 & 0 & 0 & 0 & 0 & 0 & 0 \\
\hline
\end{tabular}


Table 3. Counts for response curve pairs with downward trends for X.laevis and upward trends for X.tropicalis. The total number is 14 .

\begin{tabular}{|l|l|l|l|l|l|l|l|l|l|}
\hline & \multicolumn{9}{|c|}{ X.tropicalis } \\
\hline X.laevis & flat & lineU & tranU & hocU & sigU & impU & stepU & imp+U & step+U \\
\hline flat & 0 & 0 & 0 & 0 & 0 & 0 & 0 & 0 & 0 \\
\hline lineD & 0 & 0 & 0 & 0 & 0 & 0 & 0 & 0 & 0 \\
\hline tranD & 0 & 0 & 0 & 0 & 0 & 0 & 0 & 0 & 0 \\
\hline hocD & 0 & 0 & 0 & 0 & 0 & 0 & 0 & 0 & 0 \\
\hline sigD & 0 & 0 & 0 & 1 & 1 & 0 & 0 & 0 & 0 \\
\hline impD & 0 & 4 & 0 & 0 & 8 & 0 & 0 & 0 & 0 \\
\hline stepD & 0 & 0 & 0 & 0 & 0 & 0 & 0 & 0 & 0 \\
\hline impD+ & 0 & 0 & 0 & 0 & 0 & 0 & 0 & 0 & 0 \\
\hline stepD+ & 0 & 0 & 0 & 0 & 0 & 0 & 0 & 0 & 0 \\
\hline
\end{tabular}

Table 4. Counts for response curve pairs with upward trends for X.laevis and downward trends curves for X.tropicalis. The total number is 16 .

\begin{tabular}{|l|l|l|l|l|l|l|l|l|l|}
\hline & \multicolumn{9}{|c|}{ X.tropicalis } \\
\hline X.laevis & flat & lineD & tranD & hocD & sigD & impD & stepD & imp+D & step+D \\
\hline flat & 0 & 0 & 0 & 0 & 0 & 0 & 0 & 0 & 0 \\
\hline lineU & 0 & 0 & 0 & 0 & 1 & 2 & 0 & 0 & 0 \\
\hline tranU & 0 & 0 & 0 & 0 & 0 & 0 & 0 & 0 & 0 \\
\hline hocU & 2 & 0 & 0 & 0 & 0 & 0 & 0 & 0 & 0 \\
\hline sigU & 0 & 0 & 0 & 0 & 0 & 9 & 0 & 0 & 0 \\
\hline impU & 0 & 1 & 0 & 0 & 1 & 0 & 0 & 0 & 0 \\
\hline stepU & 0 & 0 & 0 & 0 & 0 & 0 & 0 & 0 & 0 \\
\hline imp+U & 0 & 0 & 0 & 0 & 0 & 0 & 0 & 0 & 0 \\
\hline step+U & 0 & 0 & 0 & 0 & 0 & 0 & 0 & 0 & 0 \\
\hline
\end{tabular}

Table 5. Counts for response curve pairs that are upward trends for both X.laevis and X.tropicalis. The total number is 1,052 .

\begin{tabular}{|l|l|l|l|l|l|l|l|l|l|}
\hline & \multicolumn{10}{|c|}{ X.tropicalis } \\
\hline X.laevis & flat & lineU & tranU & hocU & sigU & impU & stepU & imp+U & step+U \\
\hline flat & 0 & 0 & 0 & 0 & 0 & 0 & 0 & 0 & 0 \\
\hline lineU & 0 & 47 & 0 & 14 & 146 & 0 & 1 & 0 & 0 \\
\hline tranU & 0 & 0 & 0 & 0 & 0 & 0 & 0 & 0 & 0 \\
\hline hocU & 2 & 4 & 0 & 30 & 13 & 1 & 0 & 0 & 0 \\
\hline sigU & 0 & 20 & 0 & 73 & 694 & 3 & 3 & 0 & 0 \\
\hline impU & 0 & 0 & 0 & 0 & 0 & 1 & 0 & 0 & 0 \\
\hline stepU & 0 & 0 & 0 & 0 & 0 & 0 & 0 & 0 & 0 \\
\hline imp+U & 0 & 0 & 0 & 0 & 0 & 0 & 0 & 0 & 0 \\
\hline step+U & 0 & 0 & 0 & 0 & 0 & 0 & 0 & 0 & 0 \\
\hline
\end{tabular}




\section{Discussion}

Our algorithm allows researchers to investigate heteromorphy, heterochrony, and heterometry of biologically relevant response curves in comparative gene expression studies. When the RPE is near a threshold, model selection can be ambiguous. For example some step and impulse curves are similar to sigmoid curves when the RPE for the sigmoid curve is close to the threshold for selecting the sigmoid curve. Also the distinction between hockey and sigmoid curves is not clear when the slope of the sigmoid curve at either the beginning or end is near the threshold for steady state determination. Therefore, when using this algorithm investigators should also examine the plots of the fitted curves.

To investigate how well our algorithm reduces overfitting (in the frog data), we also investigated polynomial models (with degrees three, five and seven) in addition to biologically relevant models. Because polynomial models have little biological rationale, there is no information in the responses at fitted times that is inherently relevant to the responses at evaluation times. For example a polynomial of degree seven would perfectly fit seven points, but that says little about how well the polynomial would interpolate or extrapolate to the evaluation points. Hence an algorithm that avoids overfitting would preferentially select biologically relevant response curves over polynomial response curves. This was, in fact, the case. We found that when we also fit polynomial curves, the algorithm yielded the same distribution of biologically relevant response curve pairs (Tables 2-5) as when the polynomial models were excluded.

With modifications, it may be possible to reasonably apply this method to fewer than 14 time points. We used seven points so we could fit the seven parameters in the generalized double sigmoid model and used the remaining seven points spread evenly over the time range for evaluation. One approach for using fewer points is to simply not fit the generalized double sigmoid so that the similar double sigmoid is the most complex model investigated. Because the double sigmoid model involves six parameters, we would only need six time points for model fitting. A second approach, which can be used in conjunction with the first approach, is to use fewer evaluation points spread over the range of values at the "cost" of less information for discriminating between model fits.

To implement our algorithm we developed a set of Mathematica [14] packages called MFit. MFit requires the following input: (i) a matrix of responses for setting with rows corresponding to genes and columns corresponding to values of the varying condition (e.g., times); (ii) a list names of genes; (iii) a list of gene identification numbers; (iv) a list of times; (iv) names of time varying condition for labeling the horizontal axis; (iv) name of response for labeling the vertical axis, (iv) names of the two scenarios for labeling the top of the plot; (v) shortened form of names of the two scenarios for files for storing parameter estimates. The MFit output includes: (i) summary tables; (ii) lists of genes classified by heteromorphy, heterochrony, and heterometry for biologically relevant curves; and (iii) plots of response curves (for example Figures 2-5). The MFit program is freely available at http://prevention.cancer.gov/programs-resources/groups/b/software/mfit. The MFit program can be applied to any comparison of serial gene expression responses in two settings. The program has options for fitting the generalized double sigmoid model as the most complex model (recommended with at least 14 time points) or the double sigmoid as the most complex model (recommended with at least 12 time points). 


\section{Acknowledgments}

SGB was supported by the National Institutes of Health. The author thanks Leonid Peshkin for providing the data. This paper is published in accordance with the NIH Publication Policy.

\section{Conflicts of Interest}

The author declares no conflict of interest.

\section{References}

1. Yanai, I.; Peshkin, L.; Jorgensen, P.; Kirschner, M.W. Mapping gene expression in two Xenopus species: Evolutionary constraints and developmental flexibility. Dev. Cell 2010, 20, 483-496.

2. Chechik, G.; Koller, D. Timing of gene expression responses to environmental changes. J. Comput. Biol. 2009, 16, 279-290.

3. Sivriver, J.; Habib, N.; Friedman, N. An integrative clustering and modeling algorithm for dynamical gene expression data. Bioinformatics 2011, 27, i392-i400.

4. Bost, B.; Veitia, R.A. Dominance and interloci interactions in transcriptional activation cascades: Models explaining compensatory mutations and inheritance patterns. BioEssays 2014, 36, 84-92.

5. Moore, A. On the fundamental importance of non-linear responses. BioEssays 2014, 36, 3-4.

6. Bar-Joseph, Z.; Gitter, A.; Simon, I. Studying and modelling dynamic biological processes using time-series gene expression data. Nat. Rev. Genet. 2012, 13, 552-564.

7. Bretz, F.; Pinheiro, J.C.; Branson, M. Combining multiple comparisons and modeling techniques in dose-response studies. Biometrics 2005, 61, 738-748.

8. Ernst, J.; Nau, G.J.; Bar-Joseph, Z. Clustering short time series gene expression data. Bioinformatics 2005, 21, i159-i168.

9. Lin, D.; Shkedy, Z.; Burzykowski, T.; Aerts, M.; Gohlmann, H.W.H.; De Bondt, A.; Perera, T.; Geerts, T.; van den Wyngaert, I.; Bijnens, L. Classification of trends in dose-response microarray experiments using information theory selection methods. Open Appl. Informat. J. 2009, 3, 34-43.

10. Peddada, S.D.; Lobenhofer, E.K.; Li, L.; Afshari, C.A.; Weinberg, C.R.; Umbach, D.M. Gene selection and clustering for time-course and dose-response microarray experiments using order-restricted inference. Bioinformatics 2003, 19, 834-841.

11. Forster, M.R. Key concepts in model selection: performance and generalizability. J. Math. Psychol. 2000, 44, 205-231.

12. Burnham, K.P.; Anderson, D.R. Multimodal inference: Understanding AIC and BIC in model selection. Socio. Meth. Res. 2004, 33, 261-304.

13. Fitzpatrick, S. Simplicity in the Philosophy of Science. Internet Encylopedia of Philosophy. Available online: http://www.iep.utm.edu/simplici/ (accessed on 12 November 2013).

14. Mathematica, version 8.0; Wolfram Research, Inc.: Champaign, IL, USA, 2010.

(C) 2014 by the authors; licensee MDPI, Basel, Switzerland. This article is an open access article distributed under the terms and conditions of the Creative Commons Attribution license (http://creativecommons.org/licenses/by/3.0/). 